\title{
Short Communication \\ Abnormal morphology of Klebsiella pneumoniae in Respiratory Samples: A microscopic observation
}

\section{Dr Namita J aggi, Dr A parna Pandey* and Dr A shima J ain}

Department of Microbiology; Laboratory Services \& Infection Control, Artemis Hospital, Sector 51, Gurugram- 122001

\begin{abstract}
Klebsiella pneumoniae is one of the most common causative pathogen for respiratory infections, meningitis, urinary tract infections, enteritis and septicemia. Aberrant forms of this bacteria and some other gram negative bacteria like Escherichia coli etc. have been reported mostly in samples of patients on antibiotics. Here, we report 2 patients with respiratory illnesses, with prior antibiotic exposure history showing filamentous structures with central enlargement and capsular halo on gram stain and Klebsiella pneumoniae was grown in cultures of both respiratory specimens.
\end{abstract}

Keywords: Aberrant forms, Klebsiella pneumoniae, Gram Staining, Gram negative filaments

\section{Introduction}

Klebsiella pneumoniae is capsulated, gram-negative, short and stout bacilli. It is one of the most common causative pathogen for respiratory infections, meningitis, urinary tract infections, enteritis and septicemia. Abnormal forms of bacteria have been reported in cultures of specimens such as blood, cerebrospinal fluid, pleural fluid, and sputum samples which were obtained from patients who were receiving antibiotic therapy [1]. Here, we report an aberrant morphology of Klebsiella pneumonia observed in gram staining of respiratory samples of two patients.

\section{C ase R eports}

Case 1: A 75-year-old gentleman, a known case of diabetes mellitus and hypertension was admitted in emergency with chief complains of sudden onset high grade fever $\left(\sim 103^{0} \mathrm{~F}\right)$ and

*Author for Correspondence. E-mail: draparna29@gmail.com

www. ijamicro.com 
difficulty in breathing which progressively worsened to cause altered sensorium. Ceftriaxone and vancomycin was started empirically along with other supportive measures. He had a recent history of prior ICU admission for similar complaints associated with hypoglycemia and hyponatremia. Chest X-ray was suggestive of left lower zone opacity. Blood picture showed mild leukocytosis.

Case 2: A critically ill, 85-year-old gentleman, referred to us from other hospital presented with a history of recent cardiac arrest (post-CPR), difficulty in breathing associated with low grade fever $\left(100^{\circ} \mathrm{F}\right)$ on admission. This patient was already receiving some empirical treatment elsewhere along with other supportive treatment. Chest X-ray examination showed minimally increased bilateral radio opacities, relatively more on right side.

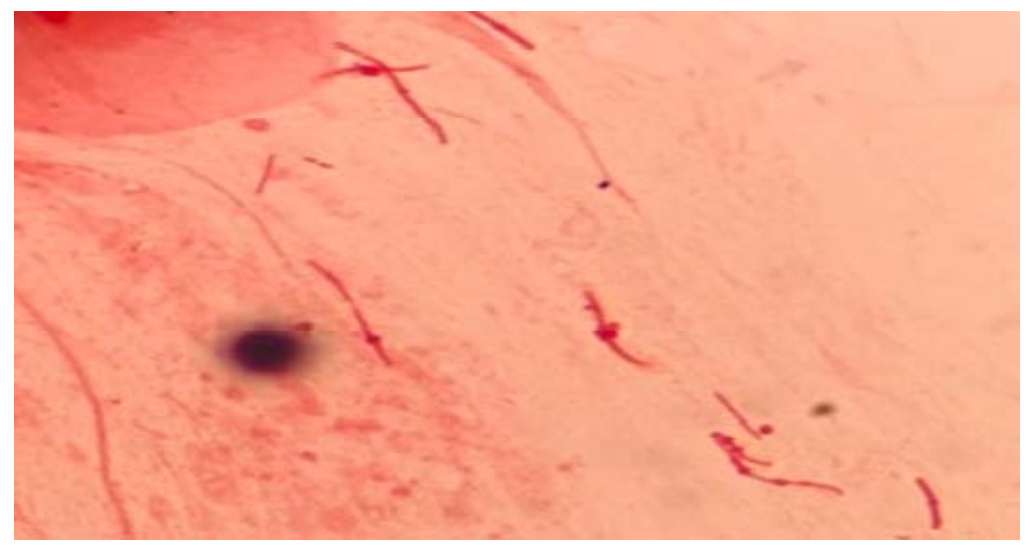

Fig.1: Klebsiella pneumoniae showing $\mathrm{G}$ ram negative filaments with central bulge morphology (G ram stain, $\times 1000$ )

Depending on clinical picture and radiological examination, respiratory infection was suspected in both the cases and hence, respiratory samples were sent for microbiological investigation. Gram staining was done for primary examination, and samples were inoculated on blood agar, Mac-Conkey agar and chocolate agar as per the lab protocols.

For the first case sputum sample was processed. The gram stain showed a numerous polymorphonuclear cells $(>25$ pus cells/high power field), few epithelial cells $(<10$ epithelial cells/high power field) and upper respiratory tract flora along with moderate number of gramnegative filamentous structures with a central enlargement (Fig.01). Some of the filaments were surrounded by an unstained zone, suggesting the presence of a capsule. Blood agar grew numerous 2-3 mm, nonhemolytic, grey white mucoid colonies with entire edges. On Mac-Conkey agar, significant growth of 2-3 mm, mucoid, lactose fermenting colonies were seen. On chocolate agar, numerous 2-3 mm, grey white mucoid colonies were seen.

For the second case, endotracheal secretion was taken and gram stain of the sample showed moderate number of polymorphonuclear cells, occasional epithelial cells, and numerous gram- 
negative filaments with central enlargement (Fig.01). Capsule was seen in some of the filaments similar to previous case.

The colonies obtained on blood agar, Mac-Conkey agar and chocolate agar were similar to the previous case. Identification and sensitivity of both the isolates were performed by both conventional laboratory methods (biochemical reactions and Kirby-Bauer method) and by automated method (Vitek-2 compact).

Isolates of both the cases were Klebsiella pneumoniae. The first one was an extended spectrum $\beta$ lactamase producer. It was sensitive to amikacin, amoxicillin/clavulanic acid, cefepime, ciprofloxacin, cefoperazone/sulbactum, gentamicin, piperacillin/tazobactum, cotrimoxazole and carbapenems and the latter was a multi-drug resistant strain with sensitivity to amikacin, gentamicin and colistin only.

\section{Discussion}

Other than Klebsiella pneumoniae, such aberrant morphological forms have also been reported in Escherichia coli, Salmonella spp. Staphylococcus aureus and Pseudomonas aeruginosa [2]. In gram negative bacteria, $\beta$-lactam antibiotics inhibit the cell wall synthesis and cause step wise cell lysis to produce morphological changes depending on the antibiotic used based on the respective cellular target [3]. Such changes have been demonstrated and confirmed by minimal inhibitory concentration (MIC) testing done with various antibiotics at different concentrations [1]. One of the reasons for the aberrant form of bacilli can either be the effect of different concentrations of antibiotics achieved in vivo due to low dose or intermittent antibiotic therapy, indicating a suboptimal antibiotic concentration at the infection site [4] or it can be due to probable coinfection with other bacteria [2]. The clinical significance of such forms is still being researched but it has been suggested that these filaments may be progenitor to spheroplast and protoplast formation, and there are few evidences of a reduced lethal effect of serum or blood on filaments. It has also been reported that spheroplasts and protoplasts have been involved in occult chronic infectious processes or recurrence of overt infection after discontinuation of antibiotic therapy [5]. Further research may also suggest a filamentous morphology being a marker for resistant strains of infection leading to a predisposition for chronic sepsis [1].

\section{Conclusion}

Microbiologists should be made aware of such aberrant appearance of various bacilli in clinical specimens to avoid confusion with fungi and other filamentous organisms. Clinicians should be explained that the appearance of these filamentous forms may indicate sub-therapeutic concentrations of antibiotic being achieved at the site of infection [2]. Also, we should strictly adhere to and reemphasize the classical practice of primary staining for all possible samples which may provide useful information about the nature of the infection and be instrumental in the decision for empirical therapy. 


\section{C onflict of Inter est}

Nil

\section{References}

1. Magnussen C. R, and Hruska J. F. 1980. "Aberrant forms of Escherichia coli in blood cultures: in vitro reproduction of an in vivo observation”. J. Clin. Microbiol.12:690-4.

2. Lorian V, Waluschka. A, \& Yehum K. 1982. "Abnormal Morphology of Bacteria in the Sputa of Patients Treated with Antibiotics”. J Clin Microbiol.Vol.16 (2): 382-6.

3. Bitterman R, Paul M, Polak D, \& Geffen Y. 2017. "Morphological changes induced by $\beta$-lactam antibiotics observed on gram staining". Clin Microbiol Infect; 23:2.

4. Saurabh K, Nag VL, Sharma A, Maurya AK, \& Hada V. 2018. "Abnormal morphological appearance of Klebsiella pneumoniae in blood culture: A microscopic observation". Indian J Pathol Microbiol 61:628-9.

5. Kinsley J, Nisheeth T, Francis G, \& Srinivas C. 2017. “Aberrant Form of Klebsiella pneumoniae in Fulminant Emphysematous Hepatitis with Polymicrobial Infection". Era Journal of Medical Research. Vol.4 [No.1]35-6. 